\title{
Latency and bit-error-rate evaluation for radio-over-ethernet in optical fiber front-haul networks
}

\author{
Sayadi, Mohammadjavad; Rodríguez, Sebastián; Olmos, Juan José Vegas; Tafur Monroy, Idelfonso
}

Published in:

Optical Switching and Networking

Link to article, DOI:

10.1016/j.osn.2017.09.002

Publication date:

2018

Document Version

Peer reviewed version

Link back to DTU Orbit

Citation (APA):

Sayadi, M., Rodríguez, S., Olmos, J. J. V., \& Tafur Monroy, I. (2018). Latency and bit-error-rate evaluation for radio-over-ethernet in optical fiber front-haul networks. Optical Switching and Networking, 27, 88-92.

https://doi.org/10.1016/j.osn.2017.09.002

\section{General rights}

Copyright and moral rights for the publications made accessible in the public portal are retained by the authors and/or other copyright owners and it is a condition of accessing publications that users recognise and abide by the legal requirements associated with these rights.

- Users may download and print one copy of any publication from the public portal for the purpose of private study or research.

- You may not further distribute the material or use it for any profit-making activity or commercial gain

- You may freely distribute the URL identifying the publication in the public portal 


\section{Author's Accepted Manuscript}

Latency and Bit-Error-Rate Evaluation for Radioover-Ethernet in Optical Fiber Front-haul Networks

Mohammadjavad Sayadi, Sebastián Rodríguez, Juan José Vegas Olmos, Idelfonso Tafur Monroy

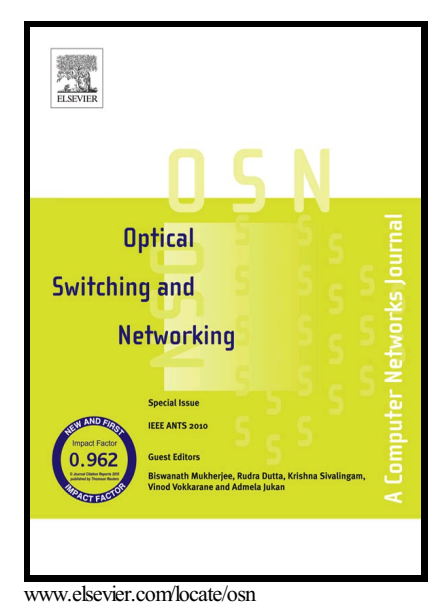

PII: $\quad$ S1573-4277(16)30189-8

DOI: $\quad$ http://dx.doi.org/10.1016/j.osn.2017.09.002

Reference: OSN456

To appear in: Optical Switching and Networking

Received date: 2 December 2016

Revised date: 10 June 2017

Accepted date: 14 September 2017

Cite this article as: Mohammadjavad Sayadi, Sebastián Rodríguez, Juan José Vegas Olmos and Idelfonso Tafur Monroy, Latency and Bit-Error-Rate Evaluation for Radio-over-Ethernet in Optical Fiber Front-haul Networks, Optical Switching and Networking, http://dx.doi.org/10.1016/j.osn.2017.09.002

This is a PDF file of an unedited manuscript that has been accepted for publication. As a service to our customers we are providing this early version of the manuscript. The manuscript will undergo copyediting, typesetting, and review of the resulting galley proof before it is published in its final citable form. Please note that during the production process errors may be discovered which could affect the content, and all legal disclaimers that apply to the journal pertain. 


\title{
Latency and Bit-Error-Rate Evaluation for Radio-over-Ethernet in Optical Fiber Front- haul Networks ${ }^{\text {ts }}$
}

\author{
Mohammadjavad Sayadi ${ }^{1,2 *}$, Sebastián Rodríguez ${ }^{1}$, Juan José Vegas Olmos ${ }^{1}$, \\ Idelfonso Tafur Monroy ${ }^{1,3}$
}

\begin{abstract}
${ }^{1}$ Department of photonics engineering, Technical University of Denmark, Oersteds Plads, building 343, 2800 Kogens Lyngby
\end{abstract}

${ }^{2}$ Technical and Vocational University, No 2, St. East Brazil, Vanak Square, Tehran, Iran

${ }^{3}$ ITMO University, 49 Kronverksky Pr., St. Petersburg, 197101, Russia

*Corresponding author. Tel.: +45-91441218. sayadi.javad@gmail.com Abstract

Nowadays several research projects are under progress to manage a soft migration toward the $5^{\text {th }}$ generation networks. Radio over Ethernet (RoE) is one of recent topics that try to have a cost efficient and independent fronthaul network. In this paper, we discuss the requirements of the 5G networks and analyze the conditions for the implementation of a RoE protocol. For this purpose we digitalize radio frames that are taken from BBU or RRH and create RoE basic frames considering all the requirements of protocol. We then encapsulate RoE basic frames into an Ethernet packet and finally experimentally evaluate this Ethernet packet as a case of study for RoE applications. The packet is transmitted through different fiber spans, measuring the BER and latency on each case. The system achieves BER values below the FEC limit and a manageable latency. These results serve as a guideline and proof of concept for applications on RoE, showing the viability of its implementation as part of the next generation of fronthaul networks.

Keywords: Radio-over-Ethernet; 5G mobile networks; Cloud radio access networks.

\section{Introduction}

Mobile data transmission is foreseen to grow 13-fold in 2017 in comparison to its volume in 2012 [1]. Also it is easily forecasted that 5th generation networks [2] will operate in a completely heterogeneous environment including different types of access technology, devices and user applications [3]. Facing with this challenge has resulted in several research works and experiments.

Cloud Radio Access Networks (C-RAN) [4,5,6] is one of recent evolutions in cellular networks to address all these challenges related to increasing the number of users and network traffic [1]. The C-RAN proposes to centralize the processing of the mobile networks, moving the base band units (BBU) from the radio access unites (RAU) to the central office. This change will simplify the RAUs, allowing the network to be more scalable [7], cost and energy efficient [8] and increase the throughput [9], but will bring new challenges with its implementation. One example is the asymmetric architecture of C-RAN in allocating functions between BBU and RRH. As Fig. 1 shows, there is only one functional layer in RRH side (Physical layer) whiles there are three functional layers in BBU side (Physical, MAC and Network layers). This asymmetric architecture creates a dependency between front-haul network and the radio access technology and increases the network burden on front-haul. This problem creates challenges is interconnecting and cooperating Base Band Units (BBU). As a way to address this problem, Radio-over-Ethernet (RoE) has been proposed to achieve a cost efficient and green solution for C-RAN challenges.

ic) 201X Published b! Science and Technolc

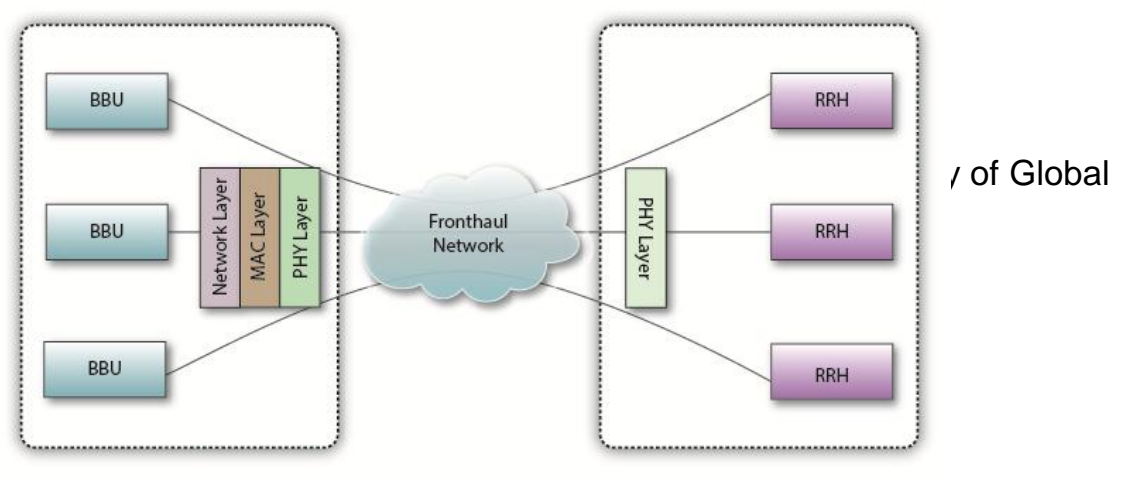


Fig. 1: Asymmetric architecture of C-RAN

In this paper, we present a general overview on Radio over Ethernet $(\mathrm{RoE})$ requirements and specifications to implement in future radio access networks. This overview later is summarized by a case of study for an experimental implementation of RoE protocol, where we evaluate the transmission of the RoE packet in terms of BER performance and latency of the process. The remainder of the paper is structured as follows: section 2 describes the architecture of RoE, section 3 describes the implementation of the RoE protocol and section 4 the experimental setup. Section 4 discusses the experimental results and finally section 5 summarizes and discusses the results.

\section{Radio over Ethernet}

Radio over Ethernet is a platform that has been standardized as IEEE 1904.3 [10]. This platform is located between RRH side and BBU side and tries to address the main challenges in C-RAN. The heart of RoE is the mapper.

As Fig- 2 shows the mapper receives radio data from RRHs or BBUs and encapsulate them into an Ethernet frame and then transmit it over an Ethernet based network. At the other side the mapper extract radio data from the received Ethernet frame and deliver them to their destinations.

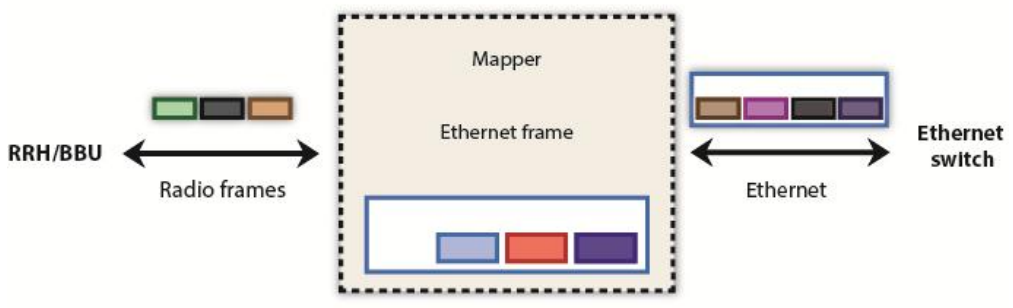

Fig. 2: Mapper of RoE and the description of its basic operation

As it was explained before, currently there is only PHY layer at RRH side and each RRH-BBU pair should use a dedicated front-haul network depend on the access technology this pair is using.

By using the RoE architecture, the mapper encapsulates all radio data types in similar Ethernet frames and the only data type is sent on front-haul network is the Ethernet frame. As the first achievement of RoE, the fronthaul network becomes independent from the radio access technology and several RRHs with different radio access technologies will be able to transfer their data over the same front-haul network.

Coordination of BBUs and cooperation between them is the other advantage of RoE, because the mapper works as an adaptor and different BBUs can easily understand each other. (Shown in Fig. 3)

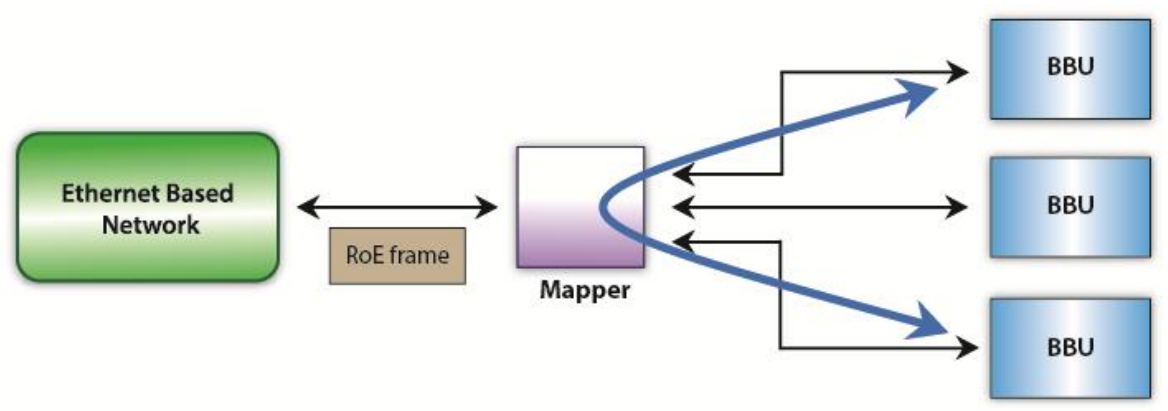


Fig. 3: Cooperating BBUs via the mapper

\section{Implemented RoE protocol}

The structure we have used for this implementation is shown in Fig. 4. In this structure, the mapper at the source side receives the radio data and put it into an Ethernet frame. By using an Ethernet switch, the Ethernet frame including the radio data is sent over the front-haul network. At the destination, the mapper gets the Ethernet frame from the switch, extract the radio data and deliver it to the final destination.

In the Ethernet frame, there is a field named Ether_Type that shows the type of data in payload. According to the values reserved for this field, we use 'E001' which is free now and can be used for RoE application. In this situation payload should be exactly 1500 Bytes because Ether_type is used for the type of data in payload and there is not any other field for the length of payload. Fig 5 shows an Ethernet frame.

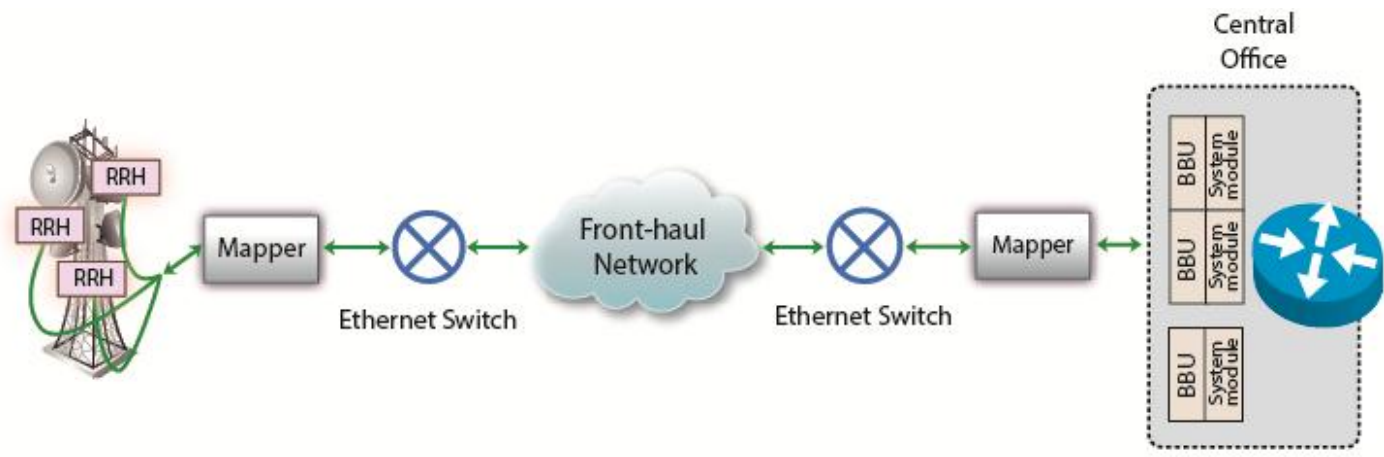

Fig. 4: RoE structure

\begin{tabular}{|c|c|c|c|c|c|c|c|}
\hline Preamble & $\begin{array}{c}\text { Start of } \\
\text { frame } \\
\text { delimiter }\end{array}$ & $\begin{array}{c}\text { Destination } \\
\text { MAC } \\
\text { address }\end{array}$ & $\begin{array}{c}\text { Source MAC } \\
\text { address }\end{array}$ & $\begin{array}{c}802.1 \text { Q tag } \\
\text { (optional) }\end{array}$ & $\begin{array}{c}\text { Ether type / } \\
\text { Length }\end{array}$ & Payload \\
\hline 7Bytes & 1 Byte & 6 CRytes & 6Bytes & 4Bytes & 2Bytes & 1500 Bytes \\
\hline
\end{tabular}

Fig. 5: Ethernet frame

The data that is received in the mapper is modulated radio signals and we are trying to encapsulate different radio data into one Ethernet frame. To manage this process we need to have a RoE sub-frame to identify and recognize each radio data from others. To simplify and optimize the protocol, this sub-frame is 20 Bytes and used for all radio data. As Fig. 6 shows, RoE sub-frame includes 16 Bytes of radio data and 4 bytes for header. This means that we can encapsulate $75 \mathrm{RoE}$ sub-frames in one Ethernet frame.

The header in RoE sub-frame has some fields to support different radio data types and approaching the goal we are targeting. The media connecting the mappers in both source and destination sides carries only Ethernet frames which are including different radio data.

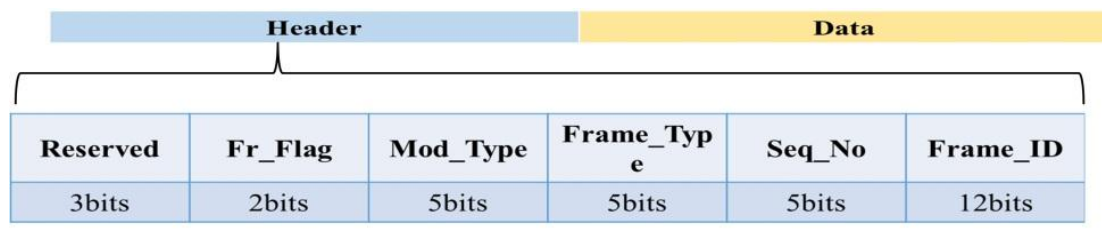

Fig. 6: RoE sub-frame

Frame_ID is a 12 bits field to identify radio frames. Starting with 0 and when it reaches 4095 , then it will be set by 0 . Each radio frame gets one Frame_ID. If we have frame larger than 16 Bytes, it has to be fragmented and all fragments get similar Frame_ID. These fragments will be differentiated by Seq_No. 


\section{ACCEPTED MANUSCRIPT}

Since the largest frame in CPRI is 324 Bytes long so Seq_no should be 5 bits long. When fragmentation is taken place, this field helps to recognize pieces of a radio frame. To manage the fragmentation and defragmentation process a 2-bit field named Fr_Flag has been used. This field shows that whole a radio data is within the payload or current frame is the first, the last or a middle part of a fragmented radio data.

Frame_Type shows the type of frame. The system is able to support several types of radio data regardless their technology and interface. We can use Type='00' in Basic Frame for invalid frames. This can helps when we have less than 75 frames in the buffer and we need to send the Ethernet frame. In this case, invalid subframes are used to fill the payload.

Mod_Type shows the type of interface or system that has produced the radio signal.

At the destination the mapper uses these fields and protocol to extract RoE sub-frames from the Ethernet frame and consequently deliver radio data to the destinations.

\section{Experimental Setup}

Fig. 7 and Fig. 8 show the setup used in the proof of principle experiments. In Fig. 7 a pulse pattern generator (PPG) is used to electrically generate the RoE packet and a 215-1 bit long pseudo-random bit sequence (PRBS15) non-return-to-zero (NRZ) signal at a data rate of $2.5 \mathrm{Gbit} / \mathrm{s}$. The signal is used to modulate the optical carrier of a small factor pluggable (SFP+) module. A variable optical attenuator (VOA) is placed for bit-errorrate (BER) measurements. The optical signal travels through different spans of standard dingle mode fiber (SSMF) to reach the receiver of the SFP+ module. The recovered electrical signal goes through a bias tee (BT) into a clock recovery system which provides both data and timing information to a bit-error-rate tester (BERT).

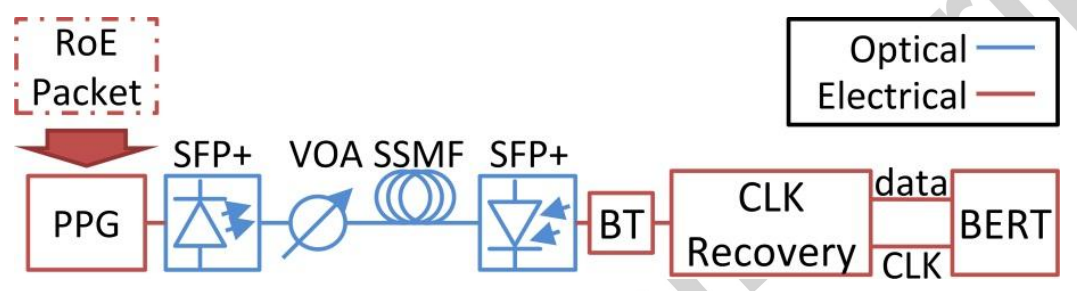

Fig. 7: RoE structure to evaluate bit error rate

In Fig. 8 we simulated an actual mapper that generates real RoE frames and injects them directly into the Network Interface Card. We used C++ to implement the mapper and used NDIS to have a driver and work directly with MAC layer. In RRH side, the mapper generate RoE frame and a Media Converter, using the same $\mathrm{SFP}+$ module, is used to transfer the data on optical fiber and reach the other Media Converter to deliver the frame to the mapper in the BBU side. Then the mapper in BBU side sends an ACK frame in RoE frame through this infrastructure. This experiment provides the round trip delay for different lengths of fiber.

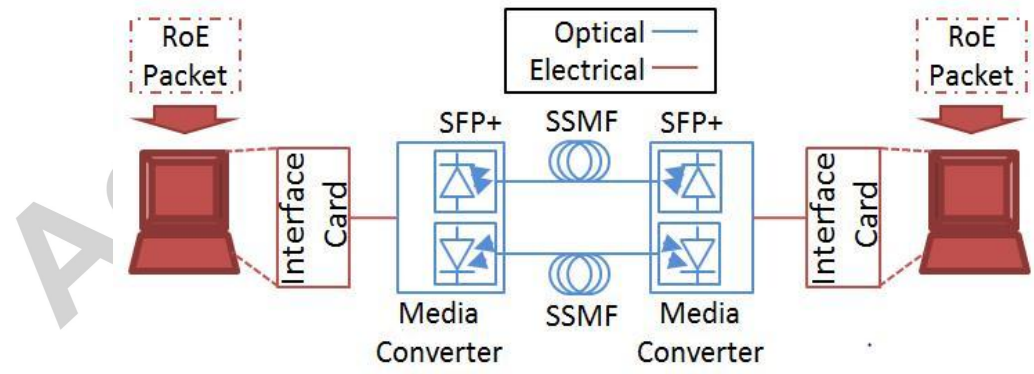

Fig. 8: RoE structure to evaluate the latency

\section{Results and Analysis}

Fig. 9 shows the BER plot of the system for two types of transmitted data: The PRBS15 and the defined RoE packet. In each case, the real-time measurement of the BER was measured for different lengths of fiber.

As depicted in Fig. 9, the system has different BER when we switch from PRBS to packet and when we want to transfer RoE packet, the system needs more optical power in comparison to PRBS. For instance to have log $(B E R)=-10$, the optical powers are -20.2 and -22.6 for packet and PRBS respectively. According to FEC limit, which is shown as a dashed line, the sensitivity of the system when we use PRBS is -27 and is -26.5 for Packet. In this scenario two lines are going to catch each other near FEC limit. 


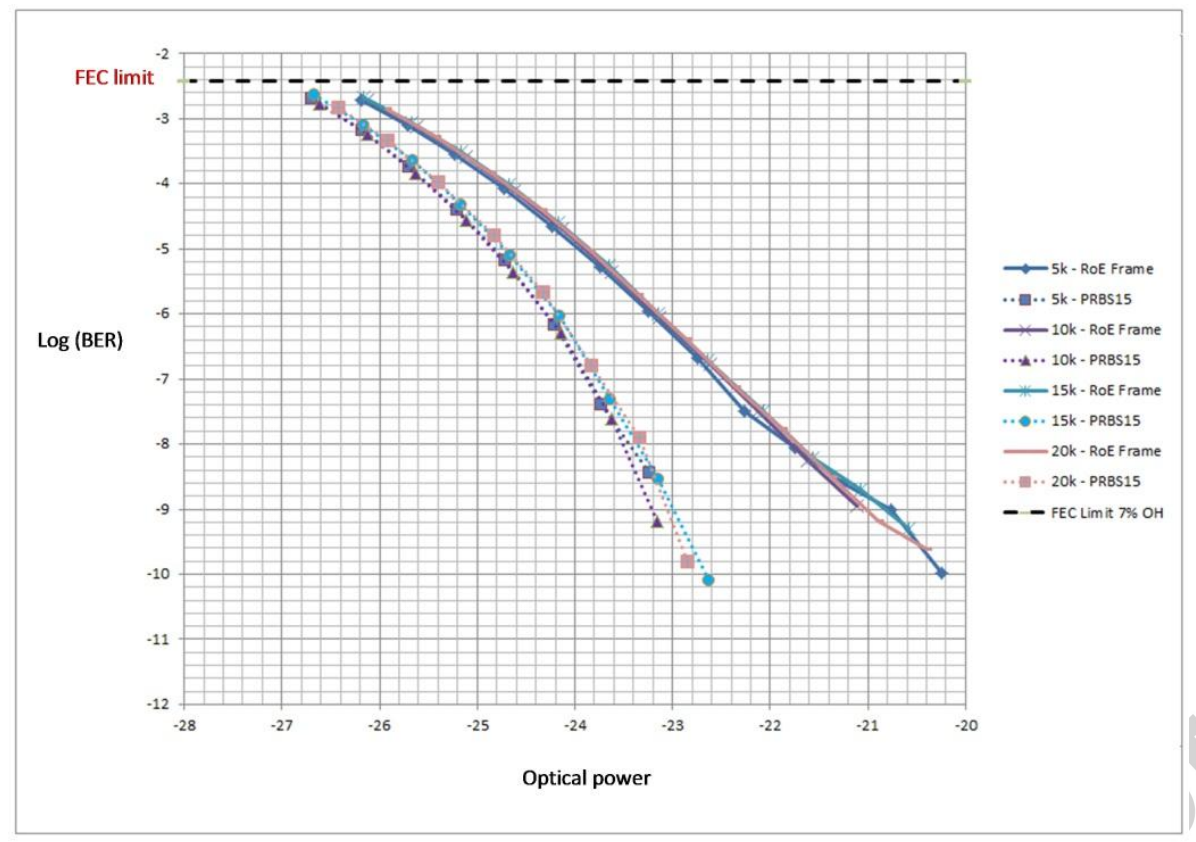

Fig. 9: BER versus optical power for RoE application

The diagram demonstrates that, the fiber length does not affect BER, since it has almost the same diagram when the fiber distance between RRH and BBU is increased.

In order to perform the latency measurements, the packet implemented and directly transmitted through the Network Interface Cards of two computers connected via two media converters with a top data rate of $100 \mathrm{Mbps}$. Fig. 10 shows the latency of round trip transmission in the experimental setup for RoE frames in an optical fronthaul network. In this figure, each line gives the round trip time for one of frame sizes between 300 Bytes and 1500 Bytes over different front-haul networks. The latency for round trip transmission not including the medium propagation delay in our experiment is varies from 1.12 to $1.18 \mathrm{~ms}$. In other words, we experienced $1.12 \mathrm{~ms}$ round trip time for an Ethernet frame including $30 \mathrm{RoE}$ sub-frame so the protocol has $37 \mu \mathrm{s}$ extra time for each radio data. The best case in the system is using the maximum size of RoE frame, because according to the size of a basic RoE frame, we can encapsulate 75 RoE sub-frames in each Ethernet frame in maximum size. As the result, we have $14 \mu$ s round trip latency for each RoE sub-frame regardless the medium propagation delay. This amount of latency corresponds to the effects of processing, transmission and all restrictions and technical features of network interface cards, media convertors and operating system. When the acceptable latency in $5^{\text {th }} \mathrm{G}$ networks is $1 \mathrm{~ms}$ our proposal has an almost negligible extra latency.

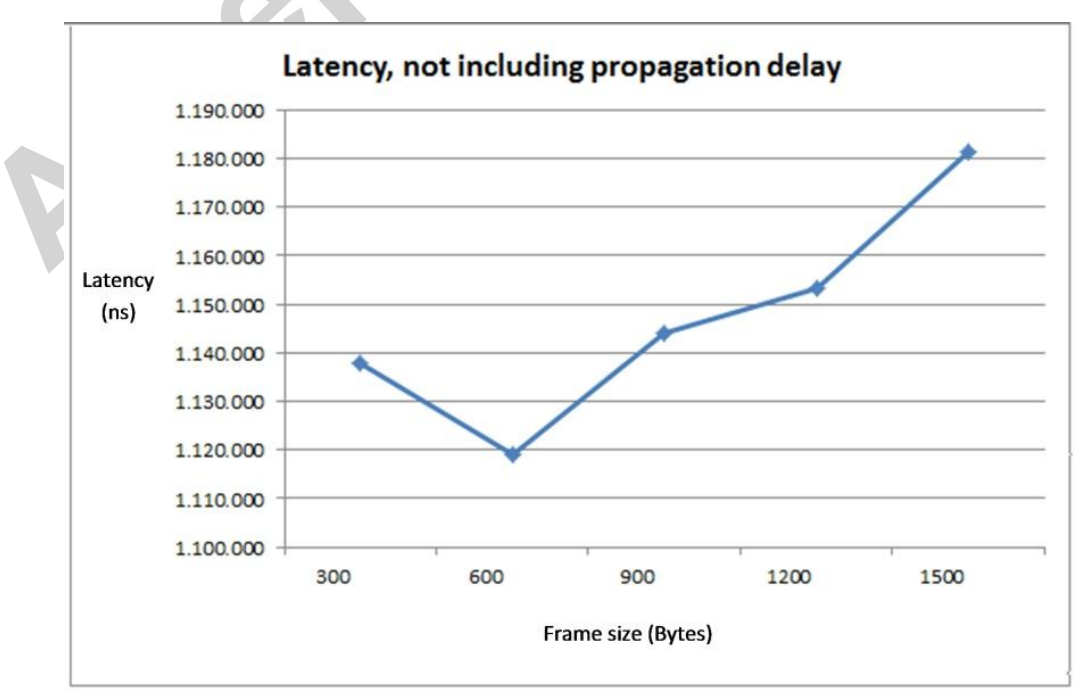

Fig. 10: System Latency, not including propagation delay 


\section{ACCEPTED MANUSCRIPT}

For the optimum case when we have 1500 bytes in payload (75 RoE sub-frames) and $14 \mu$ s of latency, including around $3 \mu \mathrm{s}$ of transmission in network interface card (Ethernet $100 \mathrm{Mbps}$ ), $9 \mu \mathrm{s}$ of data encapsulation/de-capsulation and processing in the mapper and the reminding $2 \mu \mathrm{s}$ is the effect of operating systems and other environmental events in both sides. (Fig. 11)

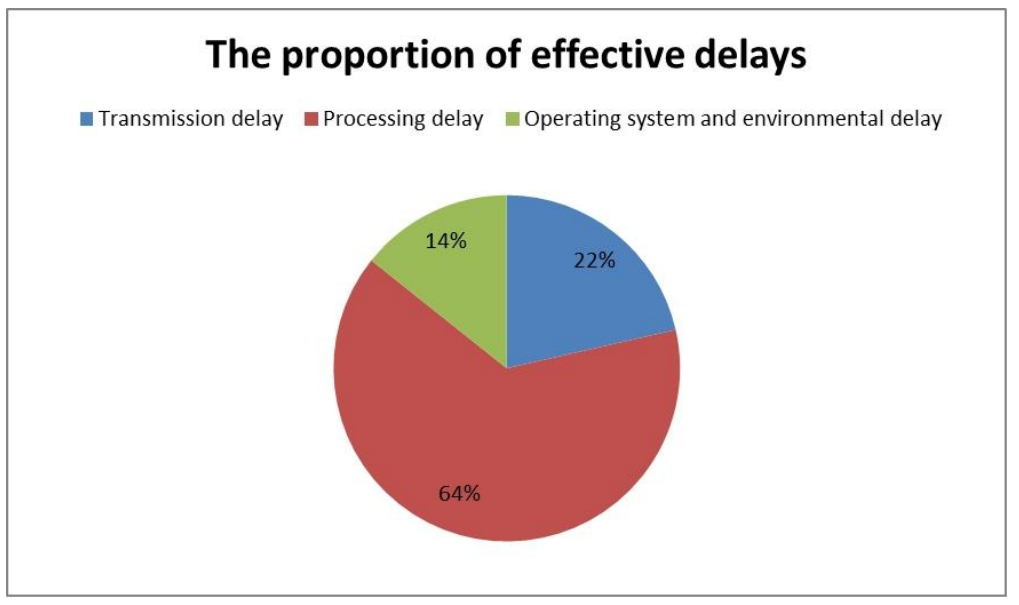

Fig. 11: The proportion of effective delays in our experiment

This experiment can be a good model for companies to develop their products to support this protocol and try to meet the latency requirement [11]. If we implement the mapper in hardware level such as FPGA, the delay related to processing delay and the delay related to the operating system will be reduced and negligible. This is technically possible because the radio data is opaque to the mapper and the system does not read its content. Also running the mapper on a switch with $100 \mathrm{Gbps}$ interfaces [12, 13] as an embedded system can help to minimize the transmission delay.

As it is shown in Fig. 12, the latency is increased when we use media convertor according to its transmission velocity and the length of data. The delay for converting and transmitting data is $0.5 \mathrm{~ms}$ when the length of frame is 300 Bytes and is $1 \mathrm{~ms}$ for the maximum size or frame.

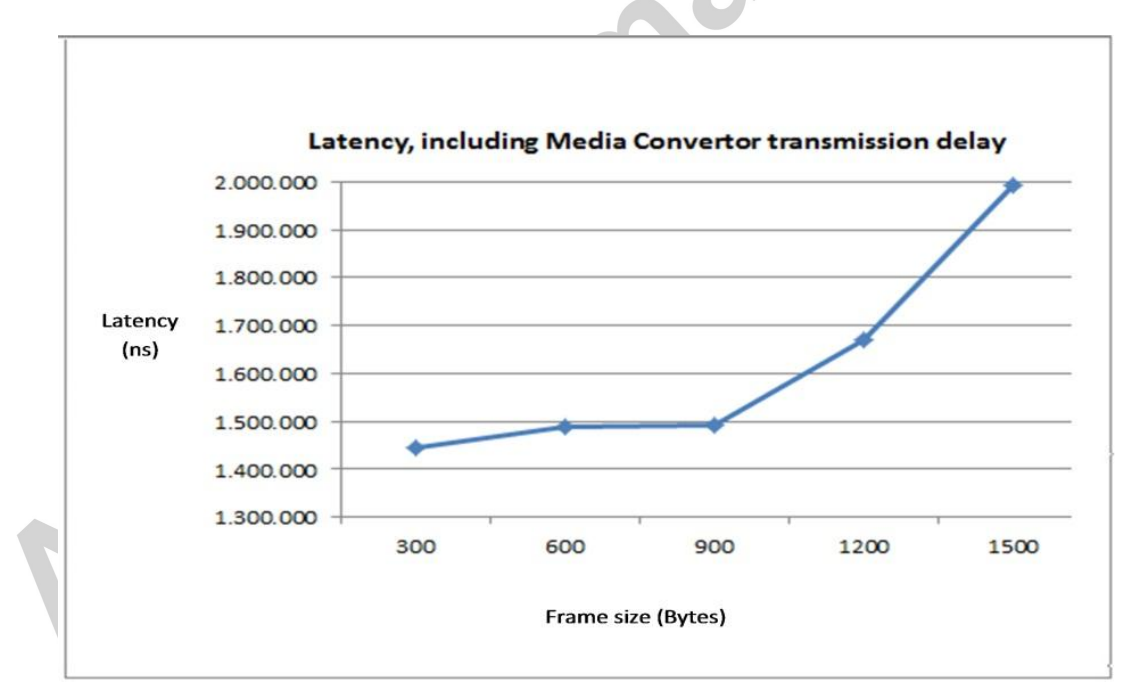

Fig. 12: System Latency, not including propagation delay

Fig. 13 shows the latency when we use different length of fiber as front-haul. In this diagram each line is the average latency over different front-haul networks for one RoE frame size. As it is shown in the diagram there are some unexpected fluctuations due to the effect of operating system and environmental delays. 


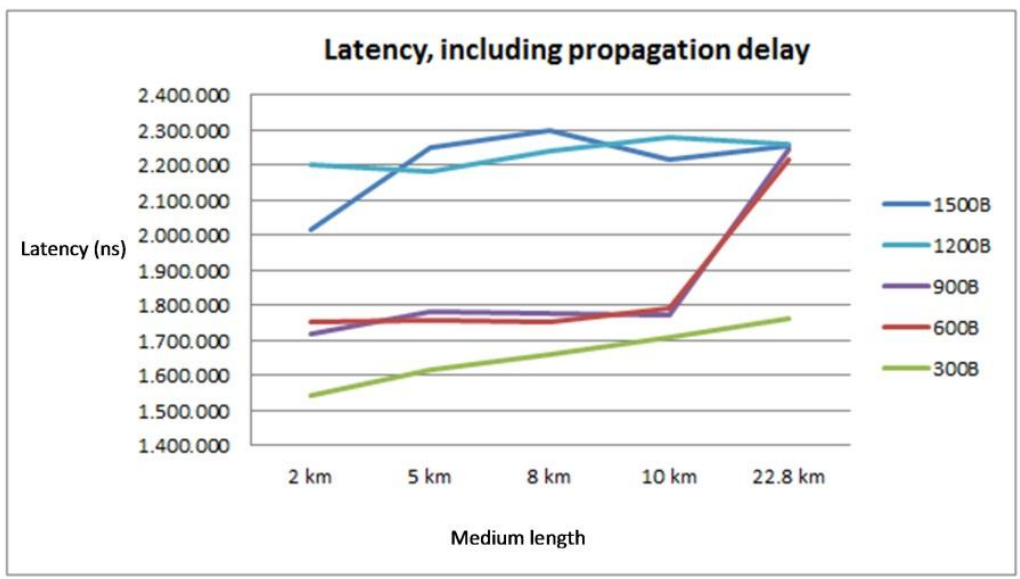

Fig. 13: Latency for RoE application

\section{Conclusion}

In this paper, we summarized the main requirements for the implementation of RoE on future access networks and demonstrated the transmission of radio data encapsulated into an Ethernet frame over an optical front-haul network. Firstly we presented a protocol for RoE applications and then implemented the protocol in an experimental setup. Mapper as the main part of the protocol was described and a RoE sub-frame was introduced according to the requirements of the protocol.

Secondly, we implemented the proposed packet in a experimental setup, to analyze the performance of the system in terms of BER and latency. The experiments depicted that our presented protocol can be implemented with BER values below the FEC limit for $7 \% \mathrm{OH}$ and negligible extra latency in the current C-RAN architecture. The results also show that although RoE applies some informational and operational header but in average the amount of the latency it increases to the $5^{\text {th }} \mathrm{G}$ networks is negligible and achieves an independent front-haul network to the access technology.

\section{Acknowledgments}

This work was partly funded by the Marie Curie FiWiN5G (grant No.642355).

\section{References}

[1] S. Jose: "Visual networking index: Global mobile data traffic forecast update, 2012-2017": USA: February 2013, Technical report.

[2] A. Checko, H. Christiansen, Y. Yan, L. Scolari, G. Kardaras, M. Berger, L. Dittmann : Cloud RAN for Mobile Networks-A Technology Overview: IEEE COMMUNICATION SURVEYS \& TUTORIALS, VOL. 17, NO. 1, FIRST QUARTER 2015.

[3] R. Hattachi, J. Erfanian: Next generation mobile networks: February 2015: white paper.

[4] C. Cicconetti: 5G radio network architecture: Radio Access and Spectrum: http://www.ict-ras.eu: white paper.

[5] Jinsong Wu Sundeep Rangan and Honggang Zhang "Green Communication" CRC Press 2013.

[6] C. M. R. Institute "C-RAN: The road towards green ran " October 2011.

[7] M. Madhavan, P. Gupta, and M. Chetlur, "Quantifying multiplexing gains in a wireless network cloud," in Proc. IEEE ICC, 2012, pp. 3212-3216.

[8] H. Jinling, "TD-SCDMA/TD-LTE evolution—Go Green," in Proc. IEEE ICCS, 2010, pp. 301-305.

[9] J. Li, D. Chen, Y. Wang, and J. Wu, "Performance evaluation of cloud-RAN system with carrier frequency offset," in Proc. IEEE GLOBECOM Workshop, Dec. 2012, pp. 222-226.

[10] IEEE P1904.3/D0.x: Draft Standard for Radio over Ethernet Encapsulations and Mappings: April 2015.

[11] C-RAN the road towards green ran: China Mobile Research Institute: October 2011: Technical report.

[12] Cisco Nexus 9516 Scaling 100G Performance to New Heights: http://networktest.com: June 2015.

[13] Transpacket, Fusion networking explained, 2012, www.transpacket.com: white paper. 